\title{
Constant-rate intravenous infusion of indocyanine green leading to high fluorescence intensity in infrared thoracoscopic segmentectomy
}

\author{
Noriyuki Misaki, MD, ${ }^{\mathrm{a}, \mathrm{b}}$ Kiichi Tatakawa, MD, ${ }^{\mathrm{a}}$ Sung Soo Chang, MD, PhD, ${ }^{\mathrm{b}}$ Tetsuhiko Go, MD, PhD, ${ }^{\mathrm{b}}$ \\ and Hiroyasu Yokomise, $\mathrm{MD}, \mathrm{PhD}^{\mathrm{b}}$
}

\section{ABSTRACT}

Objectives: The purpose of this study was to determine whether or not fluorescence could be increased by administering indocyanine green at a constant rate, thus stabilizing its blood concentration.

Methods: In 20 consecutive patients undergoing segmentectomy, the dominant pulmonary arteries were ligated, blocking blood in the target segment. Fluorescence intensity was then observed using different indocyanine green administration methods under infrared thoracoscopy. Intravenous administration of indocyanine green, via a syringe pump at a rate of $12.5 \mathrm{mg} / \mathrm{min}$, was defined as the constant rate group. The bolus group was defined by a 5 - $\mathrm{mg}$ indocyanine green rapid intravenous injection. The fluorescence intensity was compared at the time of maximum fluorescence and 2 minutes after fluorescence initiation.

Results: At maximum staining, the fluorescence intensity of the normal blood flow area was brighter in the constant rate group (median, 184.2; interquartile range, 170.2-200.1) compared with the bolus group (median, 122.3; interquartile range, 87.3-144.7; $P=.0003$ ). The fluorescence of the normal blood flow was retained even after 2 minutes. There was no difference in the fluorescence intensity of the ischemic segments.

Conclusions: The constant rate method showed brighter and better fluorescence than the bolus injection, without an increase in the dose. The contrast between adjacent segments was clear, facilitating the differentiation of the areas. (JTCVS Techniques 2020;3:319-24)

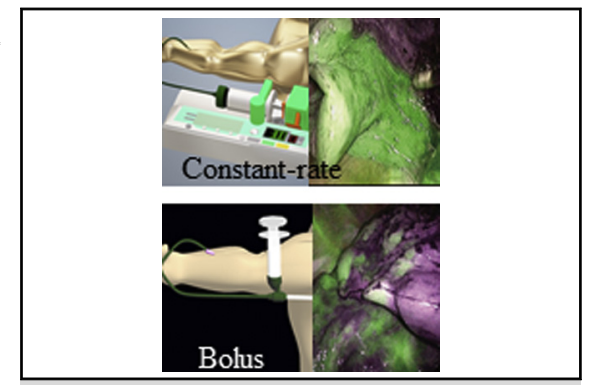

Clear fluorescence can be obtained by a constant rate injection of indocyanine green.

\section{CENTRAL MESSAGE}

Indocyanine green should be administered intravenously at a constant rate for intersegmental line observation with an infrared thoracoscope.

\section{PERSPECTIVE}

The conventional rapid infusion method of indocyanine green occasionally causes problems with the fluorescence of normal blood flow under infrared thoracoscope; this can be improved using the constant rate method.

See Commentaries on pages 325 and 327.
From the a Department of General Thoracic Surgery, Takamatsu Municipal Hospital, Takamatsu City, Kagawa Prefecture, Japan; and ${ }^{b}$ Department of General Thoracic Breast and Endocrinological Surgery, Kagawa University, Takamatsu City, Kagawa Prefecture, Japan.

Received for publication April 1, 2020; revisions received April 1, 2020; accepted for publication May 4, 2020; available ahead of print May 11, 2020.

Address for reprints: Noriyuki Misaki, MD, Department of General Thoracic Surgery, Takamatsu Municipal Hospital, 847-1 Bushozan-cho Kou, Takamatsu City, Kagawa Prefecture, Japan (E-mail: gazyucup@yahoo.co.jp).

2666-2507

Copyright (C) 2020 The Authors. Published by Elsevier Inc. on behalf of The American Association for Thoracic Surgery. This is an open access article under the CC BY-NCND license (http://creativecommons.org/licenses/by-nc-nd/4.0/).

https://doi.org/10.1016/j.xjtc.2020.05.001 $\checkmark$ Video clip is available online.

Segmentectomies are essential procedures used in patients with primary lung cancer, metastatic lung tumors, infectious diseases such as aspergillus, nontuberculous mycobacterial diseases, and benign tumors. ${ }^{1}$ The segmental line cannot be visualized from the surface, and identification of the intersegmental line is one of the challenges of segmentectomy. There are various reports on how to identify this area. Interregional identification performed in the aerated collapse line $^{2,3}$ is a common method; however, it is necessary to 


\section{Abbreviations and Acronyms \\ $2 \mathrm{M}=2$ minutes after the injection \\ $\mathrm{ICG}=$ indocyanine green \\ Max $=$ maximum}

return a portion of the aeration to the collapsed lung. When performing video-assisted thoracoscopic surgery, there is concern that the field of view will be lost. Furthermore, it is difficult to adapt for patients with severe emphysema due to the problem of air passing through the cone hole. Marking the lung surface with a pigment under a bronchoscope $^{4}$ requires preparations for manipulation with a bronchoscope other than surgery. As for injecting pigment into the bronchi, ${ }^{5,6}$ it is difficult to approach the bronchus, and the expanded lung may interfere with the visual field. Moreover, it is necessary to inject the appropriate amount of dye into the target area. Among of the simplest methods of identifying the intersegmental line is to block the blood flow to the target area and administer indocyanine green (ICG) intravenously. ${ }^{7-13}$ In addition to being simple, it is the only confirmed method in which areas identified from basic research conform to anatomical areas with a high identification rate of $84 \%$ to $100 \% .^{8-12}$

ICG that is bound to proteins and lipoproteins stays in the blood vessels, and emits fluorescence. It has a half-life of 3 to 4 minutes in the initial phase and is metabolized only by the liver. ${ }^{14}$ Based on the properties of ICG, stable fluorescence should be observed over a period of time, but in some cases, the fluorescence does not exhibit, or is thin, mottled, and washed out quickly. ${ }^{8,9}$ The causes of this have been speculated but not clearly understood. ${ }^{9}$ Clamping the pulmonary vein has been reported to increase the degree of fluorescence ${ }^{13}$; therefore, the pharmacological kinetics of ICG can be presumed to be a related cause. We hypothesized that the administration of ICG at a constant rate would stabilize its blood concentration and provide strong fluorescence. There have been no previous reports of administering ICG at a constant rate. This study aimed to confirm whether fluorescence can be increased by injecting ICG at a constant rate compared with bolus injections.

\section{MATERIALS AND METHODS}

From April 2017 to August 2019, this exploratory, cross-sectional study of constant rate versus bolus ICG intravenous administration was conducted at a single center. This study was conducted in accordance with the Declaration of Helsinki, with the approval of the in-house ethics committee of Takamatsu Municipal Hospital and written explanation and consent obtained from all patients, including consent to participate and publish the findings.

\section{Patients}

Patients aged $>20$ years, with no known allergies to ICG or iodine, who underwent segmental resection, were included in this study. We established criteria to exclude cases Child-Pugh classification $\geq \mathrm{B}$ liver dysfunction, and severe heart disease (New York Heart Association functional class $>3$ ); however, no patients fit these criteria. Five cases that used a system other than the Visera Elite II (Olympus Co, Tokyo, Japan) infrared thoracoscopic camera system utilized in this study for intersegment identification were excluded. Ten consecutive patients in the bolus group were registered from April 2017 to June 2018 and 10 consecutive patients in the constant rate group were registered from July 2018 to August 2019.

\section{Preoperative Evaluation}

All patients were examined preoperatively using 64-slices/rotation computed tomography (SOMATOM Definition AS +; Siemens Healthineers AG, Erlangen, Germany). We used the 3-dimensional image analysis system, Synapse Vincent (Fujifilm Co, Tokyo, Japan), to create 3dimensional models of the pulmonary blood vessels and bronchia, and identify the dominant blood vessels and regional bronchi in the target area for reference.

\section{ICG Injection Procedure}

We dissolved $25 \mathrm{mg}$ ICG dry powder (Daiichi-Sankyo Co, Tokyo, Japan) in $10 \mathrm{~mL}$ distilled water to make a solution with a concentration of $2.5 \mathrm{mg} / \mathrm{mL}$ and aspirated into a $20 \mathrm{~mL}$ syringe. ICG was intravenously administered through an infusion pump (Terufusion syringes pump type 35; Terumo Co, Tokyo, Japan) via a peripheral route secured on the forearm ipsilateral to the surgical side. The constant rate group received an ICG solution at a rate of $300 \mathrm{~mL} / \mathrm{h}$, based on an administration dosage reported in the previous studies. ${ }^{6,7}$ A maximum of $0.3 \mathrm{mg} / \mathrm{kg}$ solution was injected. The bolus group was rapidly injected with $2 \mathrm{~mL}$ (ICG: $5 \mathrm{mg}$ of the same concentration solution).

\section{Surgical Procedure}

The operation was performed with the patient in a lateral position under general anesthesia. A 3-port video-assisted thoracoscope, with a 3- to 4-cm access port on the anterior axillary line between the fourth and fifth ribs, a 2 -cm assist port on the lower scapula between the sixth and seventh ribs, and a $2-\mathrm{cm}$ camera port on the midaxillary line between the seventh and eighth ribs, was performed. Cases with pleural adhesion were preceded by exfoliation. The pulmonary artery of the dominant portion in the target area was ligated and separated. We then switched to infrared light observation, and ICG was administered intravenously as described above. The normal blood flow was stained green, isolating the target area, which was ischemic due to the blood vessel treatment. When the intersegmental line was identified, it was marked in the soft coagulation mode of electrocautery. We used an automatic suturing device to separate the areas according to the marks. Tumor diameter, staining start time, marking time, and surgical operation were entered into the datasheet after each operation.

\section{Image Evaluation}

All surgical videos were recorded as Moving Picture Experts Group phase 2 videos. For all cases, the still images were created using VideoStudio X 10 (Corel Co, Ottawa, Ontario, Canada) at the time of maximum fluorescence (Max) and 2 minutes after the initiation of fluorescence (2M). Using ImageJ software (National Institutes of Health, Bethesda, Md), we split the colors into red, green, and blue channels via the split channel and evaluated the fluorescence expressed in an 8-bit grayscale on the green channel. Fluorescence was expressed by measuring the intensity in 256 tones, in steps from 0 to 255 . The region surrounding the normal, stained blood and the ischemic, unstained region was designated the region of interest; the respective fluorescent intensity was obtained as an average value. The evaluators (NM and KT) of the brightness of the still images were blinded; they evaluated the brightness of the normal blood flow and the ischemic parts at Max and 2M for all 20 cases. Suitability between the evaluators was statistically analyzed. 


\section{Information Collection and Evaluation}

All of the background and surgical information was collected using patient-specific datasheets. Data notation is expressed as median (interquartile range [IQR]). The Mann-Whitney $\mathrm{U}$ test was used to compare continuous variables, and Fisher exact test was used to compare categorical variables between the groups. The correlation of variables was evaluated using Spearman rank correlation coefficient. $P$ values were examined using a 2-tailed test. All statistical analyses were performed using R version 3.4.1 (R Foundation for Statistical Computing, Vienna, Austria) of the EZR ${ }^{15}$ package. All $P$ values $<.05$ were considered statistically significant.

\section{RESULTS}

There was no difference in patients' backgrounds between the 2 groups (Table 1). The bolus group received rapid intravenous administration of $5 \mathrm{mg}$ ICG in all cases. In the constant rate group, $7.8 \mathrm{mg}$ (IQR, 5.4-9.1 mg) ICG was injected using an infusion pump. There was no significant difference in the dose of ICG per body weight, which were $0.11 \mathrm{mg} / \mathrm{kg}$ (IQR, $0.09-0.13 \mathrm{mg} / \mathrm{kg}$ ) and $0.09 \mathrm{mg} / \mathrm{kg}$ (IQR, $0.08-0.09 \mathrm{mg} / \mathrm{kg}$ ) in the constant rate group and bolus group, respectively. Fluorescence was confirmed in all cases. In both groups, fluorescence was observed $33.5 \mathrm{sec}-$ onds after the start of intravenous administration. Marking was performed at 2.82 minutes (IQR, 2.61-3.06 minutes) for the constant rate group and 2.08 minutes (IQR, 1.902.95 minutes) for the bolus group. There were no differences in the pulmonary vein or bronchial separation before

TABLE 1. Patient characteristics

\begin{tabular}{lccc}
\hline \multicolumn{1}{c}{ Characteristic } & $\begin{array}{c}\text { Constant } \\
\text { rate }(\mathbf{n = 1 0})\end{array}$ & $\begin{array}{c}\text { Bolus } \\
(\mathbf{n = 1 0})\end{array}$ & $\begin{array}{c}\boldsymbol{P} \\
\text { value }\end{array}$ \\
\hline Female & 3 & 3 & 1 \\
\hline Age $(\mathrm{y})$ & $70.5(66-79)$ & $76.5(69-81)$ & .36 \\
\hline Nonsmoker & 2 & 2 & 1 \\
\hline Body surface area $\left(\mathrm{m}^{2}\right)$ & $1.62(1.52-1.74)$ & $1.61(1.51-1.73)$ & .63 \\
\hline Type of segmentectomy & & & 1 \\
Upper lobe & 4 & 5 & \\
$\quad$ S1/S2/S1+2/S3 & $1 / 1 / 1 / 1$ & $2 / 0 / 0 / 3$ & \\
Lower lobe & 3 & 3 & \\
\hline S6/S7/S8/S9+10 & $1 / 1 / 1 / 0$ & $2 / 0 / 0 / 1$ & \\
\hline Upper division & 2 & 2 & .09 \\
\hline \%VC & 107.8 & 102.8 & \\
\hline \%FEV1 & $(101.0-119.3)$ & $(92.0-106.4)$ & \\
\hline FEV1 $1.0 \%$ & 104.7 & 104.4 & .97 \\
\hline Respiratory complication & $(97.7-121.1)$ & $(91.9-111.1)$ & \\
COPD & $76.4(72.1-79.7)$ & $76.6(70.2-80.5)$ & .97 \\
\hline CPFE & 4 & 4 & 1 \\
\hline Heart vascular disease & 2 & 3 & \\
\hline Liver cirrhosis & 0 & 1 & \\
\hline V & 4 & 0 & \\
\hline
\end{tabular}

Values are presented as $\mathrm{n}$ or median (interquartile range). VC, Vital capacity, FEVI, forced expiratory volume in 1 second; $C O P D$, chronic obstructive pulmonary disease; $C P F E$, combined pulmonary fibrosis and emphysema.
ICG administration, operation time, amount of bleeding, drainage period, or length of hospital stay after surgery between the groups (Table 2).

Two evaluators evaluated 40 images and the luminance in 80 areas. The correlation between the measured luminance intensity was 0.96 , which was consistent $(P=.0003)$.

Fluorescence intensity of the normal blood flow at the time of Max fluorescence was 184.2 (IQR, 170.2-200.1) for the constant rate group and 120.3 (IQR, 87.3-144.7) for the bolus group $(P=.001)$ (Figure 1). Even at $2 \mathrm{M}$, the fluorescence in the normal blood for the constant rate group was significantly brighter, which was 188.1 (IQR, 169.0-206.7), whereas in the bolus group it was 126.6 (IWR, 76.7-152.1) $(P=.001)$ (Figure 2). On the other hand, the fluorescence intensity of the ischemic area at Max fluorescence was 67.4 (IQR, 50.1-126.6) for the constant rate group and 77.4 (IQR, 37.9-112.6) for the bolus group $(P=.97)$. At $2 \mathrm{M}$, the fluorescence in the ischemic area was 98.7 (IQR, 88.5-104.2) for the constant rate group and 75.7 (IQR, 51.5-103.8) for the bolus group, showing no significant difference $(P=.29)$ (Table 3 and Figure 3 ).

In both groups, the intersegmental lines could be marked at least 1 portion in all cases. In the constant rate group, all intersegmental lines could be visualized and marked in 9 out of $10(90 \%)$ cases, and in 1 case, the fluorescence was mottled. In the bolus group, all intersegmental lines could be visualized and marked in 3 out of $10(30 \%)$ cases, whereas the fluorescence was thin, mottled, and early washed out in 2, 2, and 3 cases, respectively (Video 1).

In both groups, the prolonged pulmonary fistula was observed in 1 case, but no other postoperative complications were observed within 30 days after surgery.

TABLE 2. Perioperative characteristics

\begin{tabular}{lccc}
\hline \multicolumn{1}{c}{ Characteristic } & $\begin{array}{c}\text { Constant } \\
\text { rate }(\mathbf{n}=\mathbf{1 0})\end{array}$ & Bolus $(\mathbf{n = 1 0 )}$ & $\begin{array}{c}\boldsymbol{P} \\
\text { value }\end{array}$ \\
\hline Tumor size (mm) & $17.5(16.1-23.8)$ & $17.5(9.1-20.0)$ & .32 \\
\hline Precutting* of vein & 5 & 6 & 1 \\
\hline Precutting* of bronchus & 4 & 3 & 1 \\
\hline ICG dose (mg/kg) & $0.11(0.09-0.13)$ & $0.09(0.08-0.09)$ & .16 \\
\hline Appearance of staining (s) & $33.5(29.3-43.8)$ & $33.5(24.8-59.0)$ & .82 \\
Marking time (min) & $2.82(2.61-3.06)$ & $2.08(1.90-2.95)$ & .44 \\
\hline Success of all marking & 9 & 3 & .02 \\
\hline Operation time (min) & $208.5(183-243)$ & $188.5(180-198.8)$ & .36 \\
\hline Bleeding (mL) & $50(39-100)$ & $50(50-50)$ & .78 \\
\hline Duration of drainage (d) & $1(1-2)$ & $1(1-2)$ & .89 \\
\hline Postoperative stays (d) & $5(4-5)$ & $5.5(3-6)$ & .65 \\
\hline Postoperative complication & 1 & 1 & 1 \\
\hline Prolong air leak ( $>5$ d) & 1 & 1 & \\
\hline Vals are pres & 1 & 1 & \\
\hline
\end{tabular}

Values are presented as $\mathrm{n}$ or median (interquartile range). ICG, Indocyanine green. *Cutting before fluorescence. 


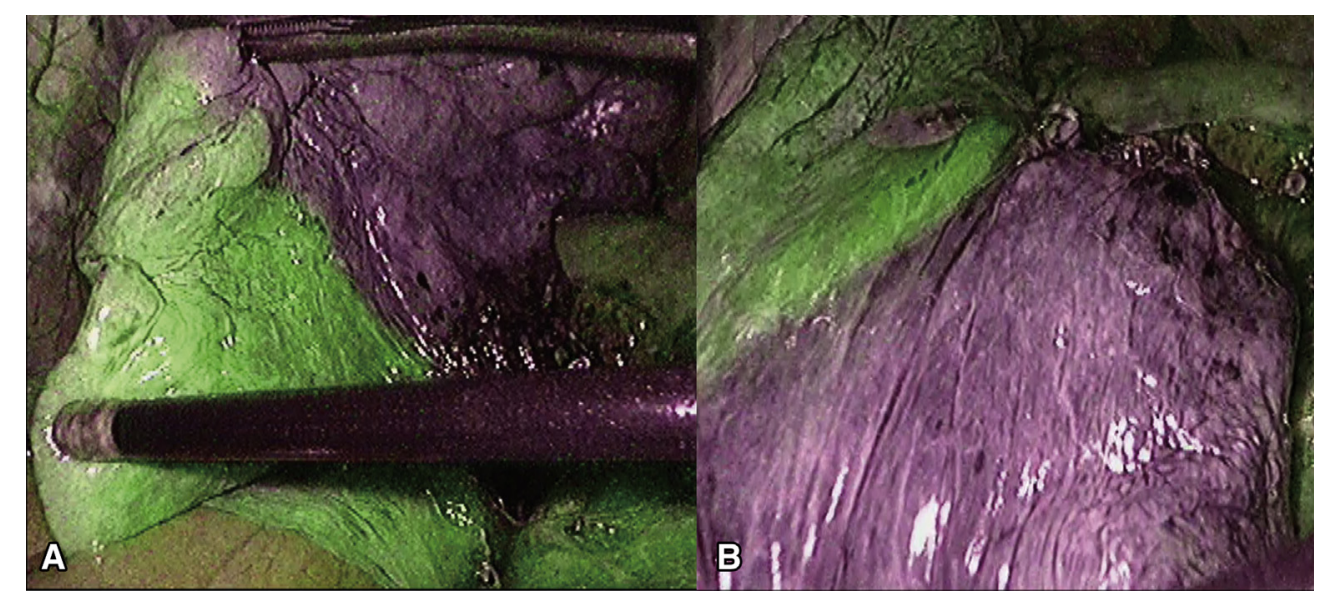

FIGURE 1. Images of a case with a constant rate. A, The whole normal blood flow area is stained, and the difference from the ischemic area can be clearly observed. B, Even after 2 minutes, the boundaries can be clearly identified.

\section{DISCUSSION}

ICG has been used in the researches of various tissues ${ }^{7-19}$; however, the method for maintaining its blood concentration at a constant rate has not been mentioned in studies so far. When examining organ performance, such as cardiac output ${ }^{16}$ and liver function, ${ }^{17}$ diluted ICG was administered as a bolus.

In cardiovascular ${ }^{18}$ and neurosurgery ${ }^{19}$ operations, blood flow is directly evaluated with a detector larger than an endoscope, making it easier to observe nonuniform concentrations. In addition, because ICG can be metabolized by the liver, it accumulates over time; thus, in segmental resections of the liver, fluorescence increases with time and is stored for several hours. ${ }^{20}$ This explains why the constant rate of ICG administration was not previously attempted for these clinical applications.
The lung is the first organ reached via the right heart system after intravenous administration, and there is not enough time for the material to diffuse. ${ }^{21}$ Over time, the material is metabolized by the liver and disappears. ${ }^{17}$ Temporarily increasing its blood concentration by bolus administration, causing a gradual decrease of fluorescence, is disadvantageous in this application. Therefore, it is beneficial to stabilize its blood concentration by intravenous administration of ICG at a constant rate.

ICG instantly binds to blood proteins and lipoproteins and becomes specifically fluorescent. It has a quenching effect, in that the fluorescence increases depending on its concentration but decreases when a certain concentration is reached. ${ }^{14,22,23}$ In aqueous solution, ICG exists as a monomer predominant at low concentrations and has fluorescent activity, but it exists as an oligomer

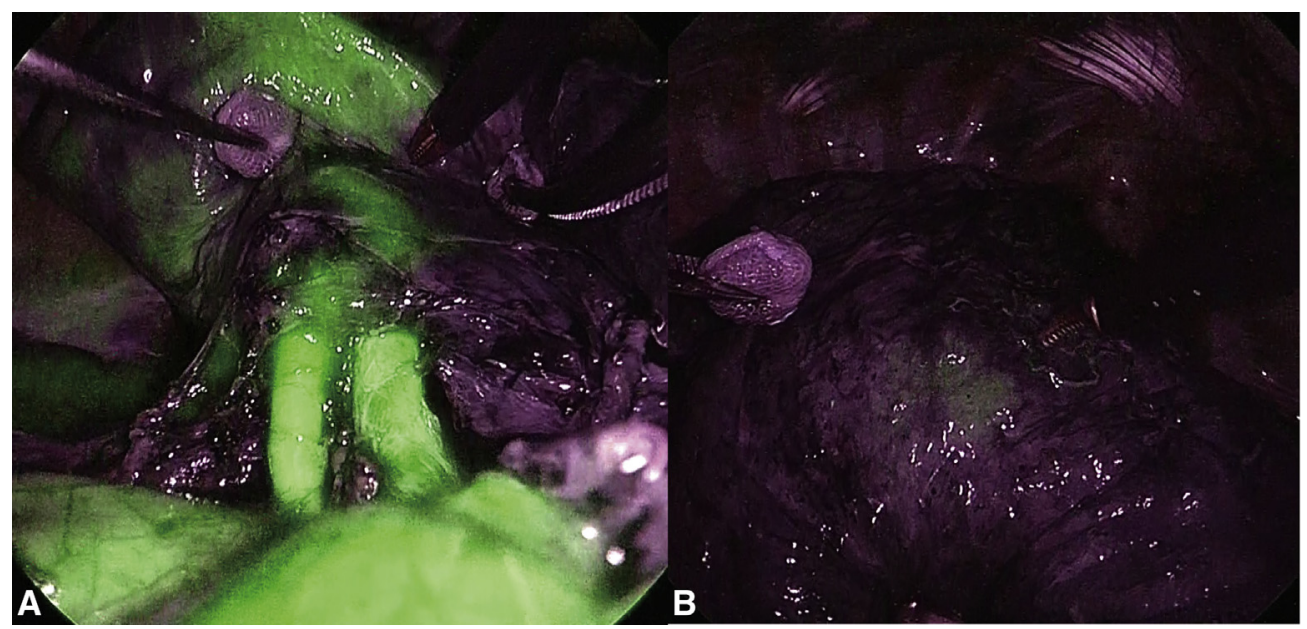

FIGURE 2. Images of a case with bolus injection. A, The normal blood flow area is stained but mottled. B, After 2 minutes, only a small part of the fluorescence remains in the unknown area. 
TABLE 3. Fluorescence among patient groups

\begin{tabular}{lccc}
\hline \multicolumn{1}{c}{ Fluorescence variable } & Constant rate group & Bolus group & $\boldsymbol{P}$ value \\
\hline Max fluorescence normal blood flow area & $184.2(170.2-200.1)$ & $120.25(87.3-144.7)$ & .0003 \\
2M fluorescence normal blood flow area & $188.1(169.0-206.7)$ & $126.6(76.7-152.1)$ & .001 \\
Max fluorescence ischemic area & $67.4(50.3-85.7)$ & $77.5(37.9-99.5)$ & .97 \\
2M fluorescence ischemic area & $98.7(88.5-104.2)$ & $75.7(51.5-103.8)$ & .29 \\
\hline
\end{tabular}

Values are presented as $\mathrm{n}$ or median (interquartile range). Max, maximum; $2 M, 2$ minutes after injection.

predominant at a high concentration, and has weak yield of fluorescence. Furthermore, ICG exists as an oligomer in a physiological saline solution. The formation of aggregate attenuates fluorescence and is observed as a quenching effect. The fluorescence properties of ICG depend on the solvent and the concentration. Compared with bolus, injecting ICG at a constant rate with a syringe pump enables the maintenance of consistent and suitable blood concentrations. ICG that exists as a monomer or binds to blood proteins may increase, and ICG that forms aggregates may decrease. It is speculated that the fluorescence intensity was maintained. The concentration showing the highest fluorescence intensity in previous reports varied from 10 to $150 \mu \mathrm{g} / \mathrm{mL},{ }^{14,23,24}$ which may be due to the effects of solvents such as plasma and whole blood and different detectors; appropriate concentration adjustment via camera systems may also be important.

This study has some limitations. It was planned as an exploratory study; therefore, it was a single-center study with a small number of subjects. However, even in 20 cases, the difference in fluorescence intensity between the groups was significant. In addition, we used a single-camera system, and there was no difference in the surgical operation or grouping of patients. The evaluation of images was blinded and measured by 2 researchers to confirm the suitability. In this way, it was confirmed that the study would be less susceptible to confounding. A single-camera system was used for this study, and in clinical application, it is necessary to consider its difference from other infrared thoracoscopic instruments. The amount, concentration, and rate of ICG administered were not examined in this study.

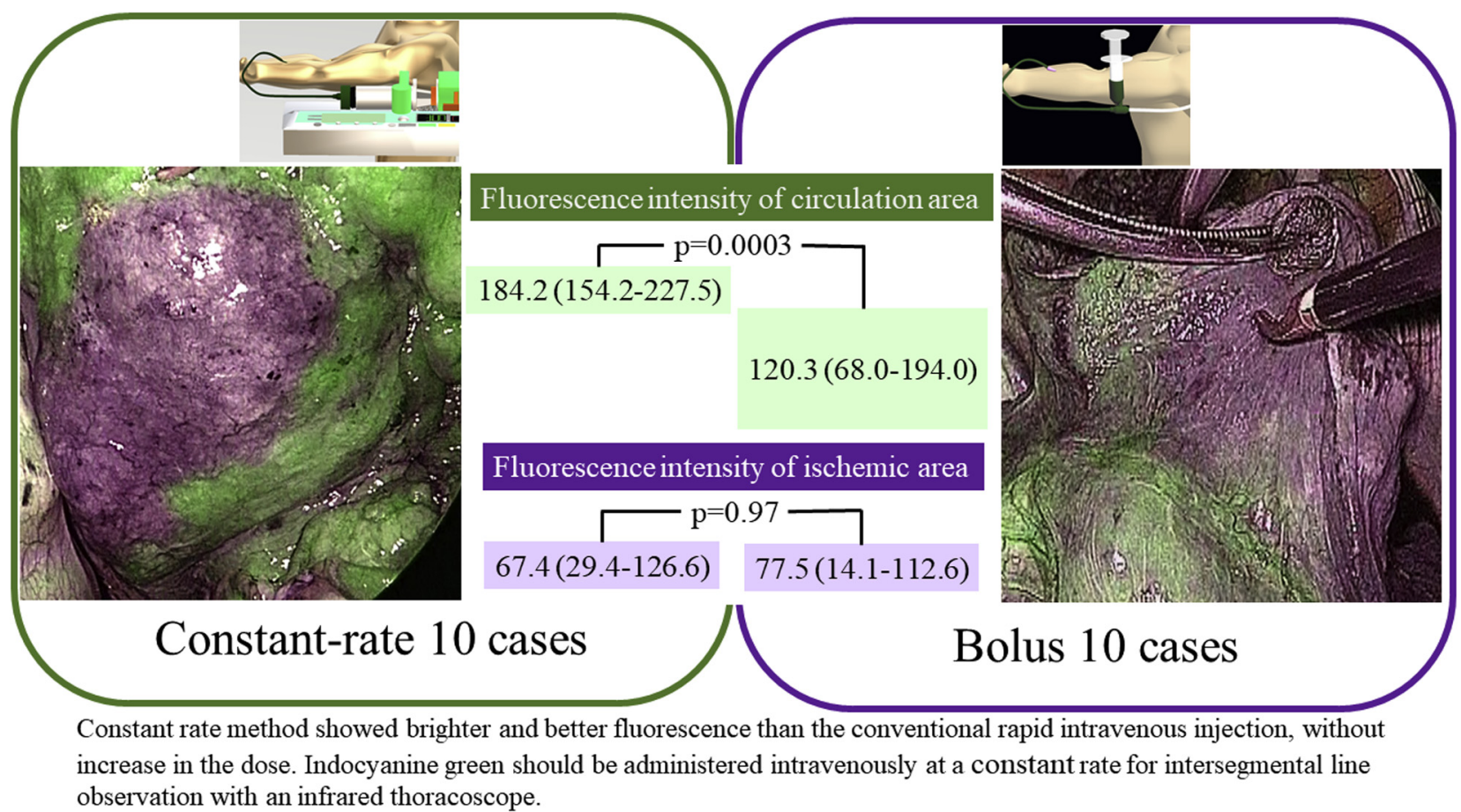

FIGURE 3. Injecting indocyanine green (ICG) at a constant rate clarifies fluorescence. The green box indicates the constant rate group, and the fluorescence intensity in the circulation area is $184.2(154.2-227.5)$, which is higher than that of the bolus group (120.3 [68.0-194.0]) (purple box) ( $P=.0003)$. There is no difference in the fluorescence intensity in the ischemic area between the 2 groups. Even in the reference image of the constant rate, a clear boundary line is obtained without increasing the amount of ICG, and the effectiveness in identifying the segmental line is confirmed. 


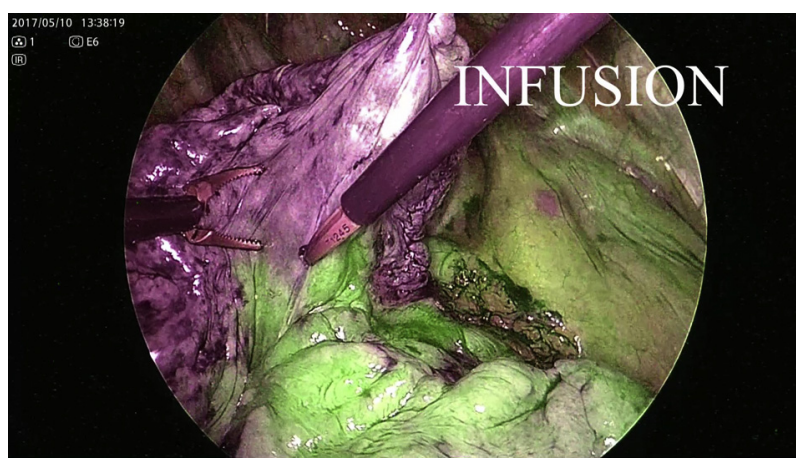

VIDEO 1. In the bolus group, the fluorescence in the first case is generally thin, in the second case is washed out fast, and in the third case is mottled. In all cases, it is more likely to be difficult to distinguish between areas. In the constant rate (infusion) group, adjacent segments be stably drawn not only in a case of simple segmentectomy but also in cases of complex segmentectomy facing a plurality of segments. Video available at: https:// www.jtcvs.org/article/S2666-2507(20)30221-2/fulltext.

\section{CONCLUSIONS}

We succeeded in obtaining fluorescence by administering ICG at a constant rate. By administering ICG intravenously at a constant rate, the fluorescence of the normal blood flow was successfully brightened, even with the same dose as in the conventional method. This method of simply administering the ICG intravenously at a constant rate allows stable segmental line identification during segmentectomy.

\section{Conflict of Interest Statement}

The authors reported no conflicts of interest.

The Journal policy requires editors and reviewers to disclose conflicts of interest and to decline handling or reviewing manuscripts for which they may have a conflict of interest. The editors and reviewers of this article have no conflicts of interest.

The authors thank Editage (www.editage.com) for providing English language editing.

\section{References}

1. Gossot D, Ramos R, Brian E, Raynaud C, Girard P, Strauss C. A totally thoracoscopic approach for pulmonary anatomic segmentectomies. Interact Cardiovasc Thorac Surg. 2011;12:529-32.

2. Okada M, Mimura T, Ikegaki J, Katoh H, Itoh H, Tsubota N. A novel videoassisted anatomic segmentectomy technique: selective segmental inflation via bronchofiberoptic jet followed by cautery cutting. J Thorac Cardiovasc Surg. 2007; 133:753-8.

3. Oizumi H, Kato H, Endoh M, Suzuki J, Watarai H, Suzuki K, et al. Port-access thoracoscopic anatomical right anterior segmentectomy. J Vis Surg. 2015;1: 2221-965.

4. Sato M, Omasa M, Chen F, Sato T, Sonobe M, Bando T, et al. Use of virtual assisted lung mapping (VAL-MAP), a bronchoscopic multispot dye-marking technique using virtual images, for precise navigation of thoracoscopic sublobar lung resection. J Thorac Cardiovasc Surg. 2014;147:1813-9.

5. Oh S, Suzuki K, Miyasaka Y, Matsunaga T, Tsushima Y, Takamochi K. New technique for lung segmentectomy using indocyanine green injection. Ann Thorac Surg. 2013;95:2188-90.

6. Sekine Y, Ko E, Oishi H, Miwa M. A simple and effective technique for identification of intersegmental planes by infrared thoracoscopy after transbronchial injection of indocyanine green. J Thorac Cardiovasc Surg. 2012;143:1330-5.

7. Misaki N, Chang SS, Igai H, Tarumi S, Gotoh M, Yokomise H. New clinically applicable method for visualizing adjacent lung segments using an infrared thoracoscopy system. J Thorac Cardiovasc Surg. 2010;140:752-6.

8. Tarumi S, Misaki N, Kasai Y, Chang SS, Go T, Yokomise H. Clinical trial of video-assisted thoracoscopic segmentectomy using infrared thoracoscopy with indocyanine green. Eur J Cardiothorac Surg. 2014;46:112-5.

9. Mun M, Okumura S, Nakao M, Matsuura Y, Nakagawa K. Indocyanine green fluorescence-navigated thoracoscopic anatomical segmentectomy. J Vis Surg. 2017;3:80

10. Guigard S, Triponez F, Bédat B, Vidal-Fortuny J, Licker M, Karenovics W. Usefulness of near-infrared angiography for identifying the intersegmental plane and vascular supply during video-assisted thoracoscopic segmentectomy. Interact Cardiovasc Thorac Surg. 2017;25:703-9.

11. Mehta M, Patel YS, Yasufuku K, Waddell TK, Shargall Y, Fahim C, et al. Near-infrared mapping with indocyanine green is associated with an increase in oncological margin length in minimally invasive segmentectomy. J Thorac Cardiovasc Surg. 2019;157:2029-35.

12. Misaki N, Chang SS, Gotoh M, Yamamoto Y, Satoh K, Yokomise H. A novel method for determining adjacent lung segments with infrared thoracoscopy. $J$ Thorac Cardiovasc Surg. 2009;138:613-8.

13. Ito A, Takao M, Shimamoto A, Shimpo H. Prolonged intravenous indocyanine green visualization by temporary pulmonary vein clamping: real-time intraoperative fluorescence image guide for thoracoscopic anatomical segmentectomy. Eur J Cardiothorac Surg. 2017;52:1225-6.

14. Desmettre T, Devoisselle JM, Mordon S. Fluorescence properties and metabolic features of indocyanine green (ICG) as related to angiography. Surv Ophthalmol. 2000;45:15-27.

15. Kanda Y. Investigation of the freely available easy-to-use software 'EZR' for medical statistics. Bone Marrow Transplant. 2013;48:452-8.

16. Calbet JA, Boushel R. Assessment of cardiac output with transpulmonary thermodilution during exercise in humans. J Appl Physiol. 2015;118:1-10.

17. Faybik P, Krenn CG, Baker A, Lahner D, Berlakovich G, Steltzer H, et al. Comparison of invasive and noninvasive measurement of plasma disappearance rate of indocyanine green in patients undergoing liver transplantation: a prospective investigator-blinded study. Liver Transpl. 2004;10:1060-4.

18. Ferguson TB Jr, Chen C, Babb JD, Efird JT, Daggubati R, Cahill JM. Fractional flow reserve-guided coronary artery bypass grafting: can intraoperative physiologic imaging guide decision making? J Thorac Cardiovasc Surg. 2013;146: 824-35.e1.

19. Riva M, Amin-Hanjani S, Giussani C, De Witte O, Bruneau M. Indocyanine green videoangiography in aneurysm surgery: systematic review and meta-analysis. Neurosurgery. 2018;83:166-80.

20. Takemura N, Kokudo N. Do we need to shift from dye injection to fluorescence in respective liver surgery? Surg Oncol. July 19, 2019 [Epub ahead of print].

21. Maseri A, Enson Y. Mixing in the right ventricle and pulmonary artery in man: evaluation of ventricular volume measurements from indicator washout curves. J Clin Invest. 1968;47:848-59.

22. Benson RC, Kues HA. Fluorescence properties of indocyanine green as related to angiography. Phys Med Biol. 1978;23:159-63.

23. Wada H, Hirohashi K, Anayama T, Nakajima T, Kato T, Chan HH, et al. Minimally invasive electro-magnetic navigational bronchoscopy-integrated nearinfrared-guided sentinel lymph node mapping in the porcine lung. PLoS One. 2015;10:e0126945.

24. Weiler M, Kassis T, Dixon JB. Sensitivity analysis of near-infrared functional lymphatic imaging. J Biomed Opt. 2012;17:066019.

Key Words: segmentectomy, indocyanine green, fluorescence, infrared thoracoscopy, constant 